\title{
Le pilotage par objectifs en Angleterre : pratiques et problématiques
}

\section{Muriel Robinson}

Traducteur : Robert Elbaz

\section{OpenEdition \\ Journals}

Édition électronique

URL : http://journals.openedition.org/ries/782

DOI : $10.4000 /$ ries.782

ISSN : 2261-4265

Éditeur

Centre international d'études pédagogiques

Édition imprimée

Date de publication : 1 décembre 2007

Pagination : 132-140

ISBN : 978-2-8542-0574-9

ISSN : $1254-4590$

\section{Référence électronique}

Muriel Robinson, «Le pilotage par objectifs en Angleterre : pratiques et problématiques », Revue internationale d'éducation de Sèvres [En ligne], 46 I décembre 2007, mis en ligne le 30 juin 2011, consulté le 02 mai 2019. URL : http://journals.openedition.org/ries/782 ; DOI : 10.4000/ries.782

Ce document a été généré automatiquement le 2 mai 2019.

(C) Tous droits réservés 


\title{
Le pilotage par objectifs en Angleterre : pratiques et problématiques $^{1}$
}

\author{
Muriel Robinson
}

Traduction : Robert Elbaz

1 L'Angleterre est sur le point d'entrer dans ce que l'on pourrait considérer comme la troisième ère de la gestion des performances. En effet, à partir du mois de septembre, tous les établissements devront avoir mis en place une politique de gestion des performances agréée par la direction et le personnel éducatif, en conformité avec les directives gouvernementales. Mon propos sera ici de présenter la littérature la plus récente sur le sujet mais sera aussi un compte rendu enraciné dans l'expérience du terrain. J'illustrerai cet article d'extraits d'un entretien approfondi que j'ai eu avec un directeur anglais chevronné du secondaire qui a lui-même expérimenté les étapes du développement que nous avons traversées lors de la mise en place du système actuel.

2 Dans un premier temps, je définirai les grandes lignes du nouveau système tel qu'il va être mis en œuvre, puis l'explorerai, ainsi que ses manifestations antérieures, afin de répondre aux questions suivantes: Comment le pilotage par objectifs et les priorités pédagogiques sont-ils liés? Comment le pilotage a-t-il finalement été accepté par les enseignants? Comment met-on en place une régulation entre établissements? Quelles transformations ce pilotage induit-il?

\section{Le système anglais}

Pour comprendre comment fonctionne le système, il est nécessaire d'avoir au préalable un aperçu de la façon dont les enseignants du primaire et du secondaire sont rémunérés en Angleterre. Il existe trois niveaux de rémunération, comme le montre le diagramme suivant : 


\section{Système de rémunération}

Échelon de directionaugmentation liée

au pilotage par objectifs

Échelon supérieur-

plus de $50 \%$ des profs-

augmentation liée

au pilotage par objectifs

\section{Premier échelon salaire-}

augmentation annuelle automatique

mais aussi pilotage par objectifs

4 Le premier niveau, celui où tout enseignant se trouve en début de carrière et où se situe environ la moitié des effectifs enseignants à un moment donné, comporte une série d'augmentations versées annuellement. Ces augmentations n'ont aucun rapport avec la gestion des performances de l'enseignant, sauf dans le cas très rare où celles-ci posent problème, auquel cas il est possible de ne pas les accorder. Partant de ce grade professionnel le plus répandu, il est possible de postuler pour le grade supérieur, où les augmentations ne sont plus automatiques mais tributaires des résultats obtenus par rapport à des critères préétablis liés au cycle annuel de gestion des performances. Enfin, pour celles et ceux qui deviennent directeurs ou adjoints de direction, il existe une échelle d'évaluation particulière, également liée aux objectifs de performances. 


\section{La hiérarchie d'évaluation}

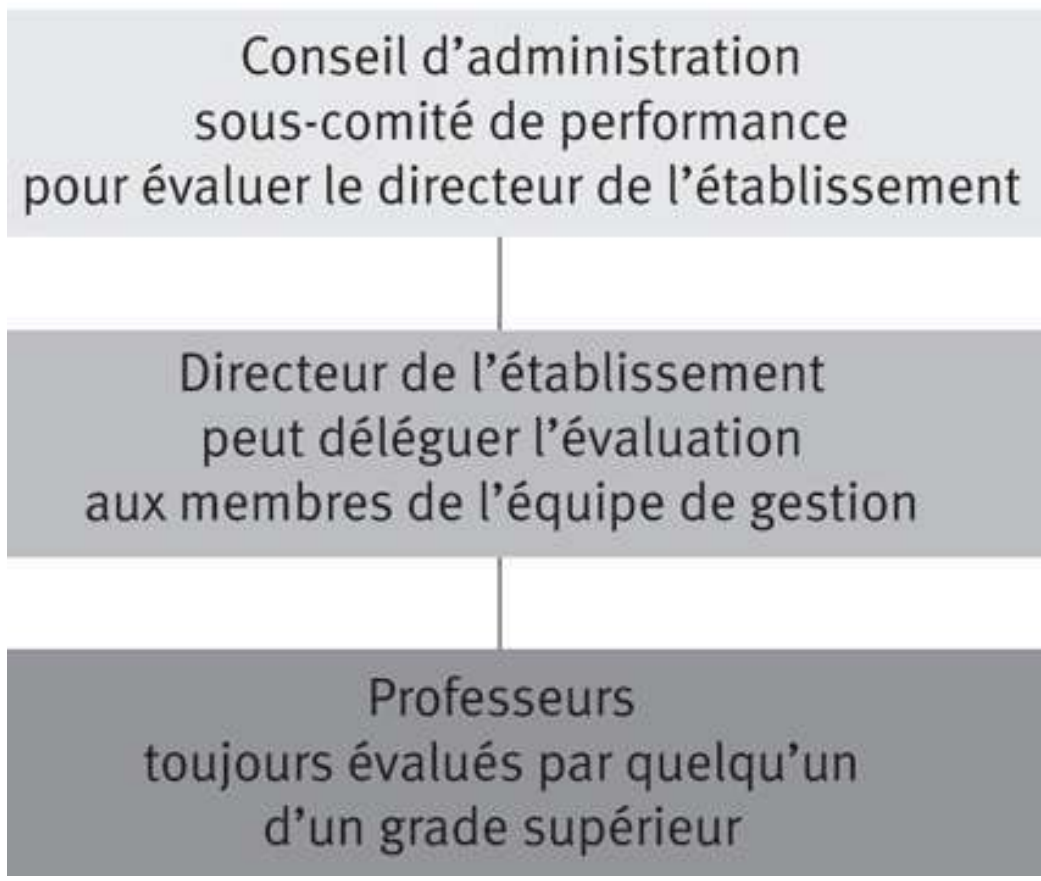

$5 \quad$ Associée à cette hiérarchie des rémunérations, il existe une hiérarchie des responsabilités en matière d'évaluation des progrès accomplis par rapport aux objectifs définis. Tout enseignant a le droit de faire évaluer ses performances par une personne d'un garde plus élevé, qui sera en principe le directeur ou le proviseur. C'est effectivement la norme dans les petites écoles primaires. Dans les établissements plus grands, ce n'est guère viable, si bien que le chef d'établissement a la possibilité de confier cette tâche au personnel d'encadrement, tout en assumant la responsabilité des évaluations assurées par ledit personnel. Les performances du chef d'établissement, elles, sont gérées par un souscomité du conseil d'administration ${ }^{2}$ appuyé par un partenaire d'amélioration scolaire ( School Improvement Partner, ou SIP), la plupart du temps un inspecteur local. Ce souscomité décide chaque année si oui ou non les performances du chef d'établissement sont suffisantes pour justifier une augmentation de salaire.

Bien qu'il y ait une relation directe entre rémunération et performance pour de nombreux enseignants, et pour l'ensemble des chefs d'établissement, il semble n'y avoir que peu de preuves venant étayer l'hypothèse que ceci ait été un élément décisif dans l'évolution du système scolaire anglais. Le chef d'établissement que j'ai interviewé confirme ce point de vue : «Je ne pense pas que ce soit un facteur déterminant. La vision du genre "Je ne suis là que pour l'argent, je vais essayer d'en faire le moins possible et de gagner le plus d'argent possible" est vraiment minoritaire, ce qui fait que ce n'est guère l'argument principal pour la plupart des gens. L'argument principal, c'est la sensation que l'on a lorsqu'on sait qu'on a fait du bon travail, ou la réaction d'un élève lorsque tout marche bien ». 


\section{Le cycle de pilotage par objectifs}

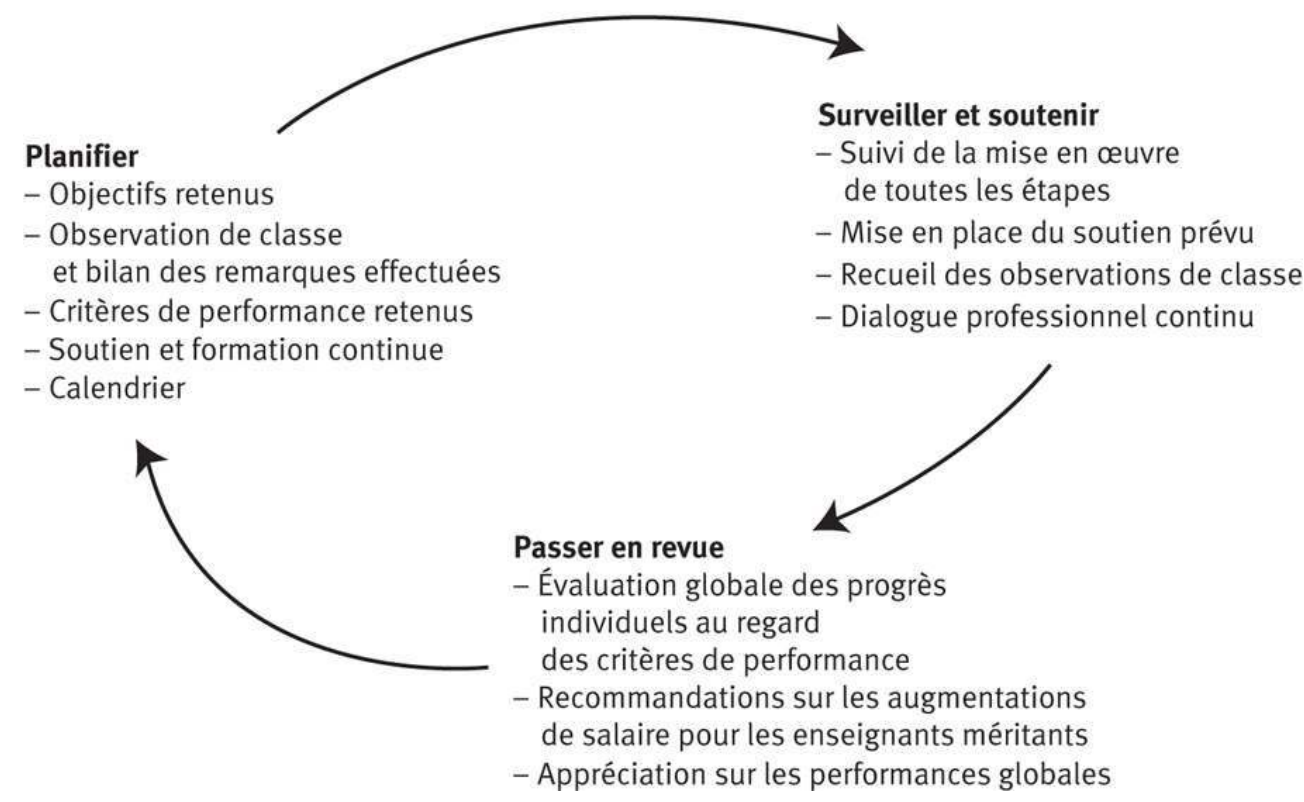

Pour l'ensemble du personnel enseignant d'un établissement, il existe un cycle annuel de gestion des performances, comme le montre le diagramme ci-dessus ${ }^{3}$ :

Bien évidemment, puisqu'il s'agit d'un cycle, ce processus permanent n'a ni commencement ni fin. Cependant, il est possible de définir un parcours annuel type pour comprendre précisément l'expérience que vit un enseignant durant l'année. Au début du premier trimestre - ou, comme c'est souvent le cas, à la fin du troisième trimestre de l'année précédente-, chaque enseignant rencontre son évaluateur pour définir en concertation une série de performances cibles fondées sur les progrès accomplis durant l'année précédente, sur les objectifs actuels de l'école et sur les aspirations de carrière de l'enseignant. Ces objectifs doivent être SMART ${ }^{4}$ : Spécifiques, Mesurables, Atteignables (Accessibles ?), Réalistes et inscrits dans le Temps. Tous les besoins de développement de l'équipe enseignante associés à ces objectifs sont ensuite identifiés, puis des stratégies pour répondre à ces besoins sont mises en place.

Durant l'année, l'enseignant rencontre son évaluateur à intervalles réguliers pour discuter des progrès et plus particulièrement des résultats des observations faites en classe, qui sont considérées comme faisant partie intégrante du processus. À la fin du cycle, une réunion sommative permet de juger les progrès accomplis par l'enseignant au cours de l'année et de répondre à d'éventuels besoins de développement. Lors d'un cycle en tous points comparable, les chefs d'établissement ont des objectifs à atteindre, définis en partenariat avec le sous-comité de gestion. Ils sont évalués en fin de cycle sur la manière dont ces objectifs ont été ou non atteints.

Afin d'assurer le bon fonctionnement de ces cycles, certains éléments significatifs doivent impérativement être réunis, comme mon chef d'établissement témoin l'a confirmé. Tout d'abord, les conditions physiques dans lesquelles ces réunions se tiennent doivent être optimales. Il est essentiel que les réunions ordinaires disposent d'un laps de temps suffisant pour se tenir et qu'elles ne soient pas réduites à des pauses-café ou à des déjeuners pris en commun. Ce n'est certes pas facile car les enseignants ont tendance à faire de la résistance dès lors que ces réunions empiètent sur leur temps face à la classe. 
Toutefois, il est quasiment impossible de traiter ces questions de manière un tant soit peu approfondie sans la possibilité de pouvoir en débattre de façon ininterrompue et prolongée. Ces réunions doivent se tenir dans un endroit isolé et confortable, téléphones portables coupés. C'est, là aussi, un idéal difficile à atteindre en milieu scolaire, souvent bruyant, où fort peu de gens disposent d'un bureau personnel.

11 Ensuite, les relations entre l'enseignant et son évaluateur doivent être bonnes. La confiance et le respect mutuels sont fondamentaux. Si d'aventure l'enseignant jugeait que son évaluateur n'a pas une bonne perception des réalités du métier, le jugement que pourrait porter cet évaluateur n'aurait pas grand poids, quand bien même il serait positif. De la même façon, si l'enseignant ne considère pas son évaluateur comme plus expérimenté ou plus compétent que lui, il doutera de la pertinence des conseils qui lui seront prodigués.

12 Enfin, il faut tendre vers un équilibre scrupuleux, de manière à ce que les objectifs définis recouvrent les besoins de l'établissement tels qu'ils apparaissent dans le projet de développement scolaire (School Development Plan) d'une part, et à ce que les besoins et les ambitions de l'individu, de son développement et de sa progression professionnels soient pris en compte, d'autre part. Ainsi, lorsque l'évaluateur et l'enseignant évalué comprennent clairement les règles et les enjeux de l'évaluation, nous créons les conditions d'une situation dans laquelle chacun trouve son compte. Les deux parties ont intérêt à ce que le cycle de gestion des performances fonctionne correctement.

13 Les modalités d'utilisation des observations faites en classe pour définir les objectifs à atteindre et les progrès accomplis au regard de ces mêmes objectifs sont également décisifs. Si l'enseignant estime que la réalité de la salle de classe est totalement déconnectée de ces objectifs, il n'aura guère confiance en ceux-ci, pas plus qu'en la personne qui les définit.

14 Voilà donc le système anglais - et il est ainsi depuis quelques années déjà. Mais quelle est son efficacité? Est-il bien accepté? Quels bénéfices peut-on en retirer en termes de réussite des élèves?

\section{Pilotage par objectifs et priorités pédagogiques : quel lien?}

15 Lorsque j'ai posé cette question à mon chef d'établissement témoin, il a identifié la relation entre l'approche actuelle de la gestion des performances et le développement scolaire comme l'un des bénéfices essentiels: «Pour ma part, je pense que l'une des caractéristiques essentielles du système de gestion des performances est le fait qu'il a été possible, grâce aux objectifs assignés dans ce cadre, de refléter véritablement l'ensemble des questions auxquelles est confrontée l'école. Il est vrai qu'au départ les objectifs étaient du genre "Bon, eh bien, qu'est-ce que vous voudriez faire ?" Ils étaient centrés autour du développement des personnels d'éducation, alors qu'ils gravitent maintenant davantage autour de réflexions tels que "Voilà les enjeux cruciaux pour améliorer l'établissement. En quoi vos objectifs personnels vont-ils y contribuer ?" ".

16 Chaque établissement scolaire se fixe des objectifs quantitatifs, étroitement associés aux résultats des examens précédents et à ceux des établissements de même type du voisinage. Comme les résultats d'examens sont publiés et accessibles, et qu'en conséquence les parents peuvent utiliser ces informations pour choisir l'école de leurs 
enfants, la pression est forte pour mener à bien ces améliorations. Les objectifs individuels des enseignants incluront ainsi également les résultats liés à la réussite de leurs élèves - par exemple que $50 \%$ d'un groupe particulier se voient attribuer un $A$, un B ou un $C$ dans une matière donnée au GSCE ${ }^{5}$. Cependant, ces objectifs viendront épauler le plan de développement scolaire de bien d'autres façons, qui relèvent d'objectifs pédagogiques. Par exemple, un enseignant pourrait se voir chargé de mettre au point un projet de travail taillé sur mesure pour un groupe d'élèves donné afin de lui permettre d'améliorer sa compréhension de tel ou tel aspect du programme. Il pourrait tout aussi bien approfondir l'utilisation par ce groupe des techniques de questionnement ou celles des activités en classe entière dans leur emploi du temps. C'est là que le lien entre observation des cours et détermination des objectifs entre en jeu. Les enseignants pourraient tout à fait demander à l'observateur de prêter plus particulièrement attention à un domaine qu'ils souhaiteraient améliorer afin d'obtenir des informations spécifiques en retour, informations qui pourraient à leur tour donner corps à ces objectifs. En d'autres termes, il existe dans le système anglais un rapport tout à fait étroit entre l'utilisation de la gestion des performances, la détermination des objectifs et le désir d'améliorer tout à la fois la pédagogie et la réussite des élèves. Sans ce rapport étroit, il aurait été bien plus difficile de mettre en place ce système.

\section{Comment le pilotage a-t-il finalement été accepté par les enseignants?}

17 L'évolution vers le système actuel s'est faite tout en douceur, ce qui explique qu'il a été bien plus facile à mettre en place que les dispositifs précédents. La toute première version, introduite à la fin des années quatre-vingt-dix, consistait en une simple évaluation annuelle et ne comportait que des objectifs faciles à atteindre, sans lien avec la rémunération. Les enseignants s'habituèrent à l'idée d'une réunion annuelle portant sur leurs projets à venir et commencèrent à en voir les avantages. Entre-temps, l'observation par les pairs fut mise en place, comme technique d'enseignement et de formation. On encouragea les enseignants à s'observer les uns les autres, à apprendre de la pratique professionnelle des autres et à fournir une précieuse information en retour à la personne qu'ils avaient observée, comme l'explique mon témoin : "Comme chef d'établissement coordinateur, l'une de mes tâches était de piloter le système d'évaluation et de le mettre en place, et je me rappelle très bien la réunion avec l'ensemble de l'équipe ainsi que la façon dont nous les avons convaincus à l'époque, en leur disant que c'était une occasion rêvée de travailler avec un collègue, de se poser et de réfléchir, d'avoir une autre paire d'yeux dans la classe pour analyser notre pratique, de pouvoir affiner notre propre façon d'enseigner, si bien que c'était davantage perçu comme de la gestion des personnels que comme de la gestion des performances. Oui, c'est bien comme ça que nous avons convaincu les collègues: "quelle merveilleuse occasion". Nous avons présenté tout ça comme étant une observation réciproque».

Dans sa version première, le système relevait d'un processus qui se situait hors de toute hiérarchie et de tout jugement. L'étape suivante a été l'introduction d'une nouvelle échelle de rémunérations située au-delà des principaux indices existants, échelle dont les enseignants pouvaient demander à bénéficier en apportant la preuve qu'ils disposaient des compétences et du savoir-faire requis, mesurés à l'aune d'une série de normes officielles et publiques. Quoique relativement rigoureux en ce qui concerne les preuves 
que les enseignants devaient apporter, cet exercice de transfert qui consistait à « franchir le seuil ", comme on le disait, a propulsé quasiment l'ensemble des postulants vers cette nouvelle échelle, avec des avantages financiers conséquents qui ne manquèrent pas de susciter de nouvelles candidatures.

Ainsi, les enseignants se familiarisèrent avec les trois ingrédients clefs du système actuel - l'observation en classe, les bilans annuels de performance et l'indexation de la rémunération aux résultats. Il fut alors relativement facile d'interconnecter ces trois éléments et de mettre en place un système de gestion des performances plus formalisé. À cette époque, les établissements bénéficiaient de rallonges budgétaires permettant de financer la formation des personnels en termes d'observation, de gestion de l'information en retour et de définition des objectifs. Ces rallonges budgétaires font maintenant partie intégrante de la dotation budgétaire habituelle des établissements, qui sont dorénavant tenus de gérer leur budget en prévoyant cette ligne de dépenses pour financer cette évaluation.

Alors que le système dit «de seuil» se mettait en place, il fut relativement facile au gouvernement de préparer les chefs d'établissement à définir leurs propres objectifs. Ils avaient la responsabilité initiale d'évaluer leurs personnels éducatifs, ce qui décidait de leur progression vers l'échelle de rémunération supérieure - bien que ce processus d'évaluation ait été modulé par des intervenants extérieurs. Il leur revenait aussi de persuader leurs personnels de l'intérêt de définir des objectifs selon les critères SMART et de mettre en place l'observation dans les classes. Le fait d'avoir leurs propres objectifs et de s'inscrire eux aussi dans ce processus de gestion des performances leur permit de montrer qu'ils faisaient eux-mêmes ce qu'ils demandaient à leurs personnels de faire. Ainsi, il ne fut guère difficile de persuader presque tous les chefs d'établissement de s'engager à appliquer ce nouveau système, comme mon témoin le dit: "Très franchement, je pense que le gouvernement et le $D f E S^{6}$ ont manœuvré avec beaucoup d'intelligence parce que, bien sûr, les objectifs de performance des chefs d'établissement ont été mis en place en même temps que les seuils, si bien qu'il aurait été difficile, pour ne pas dire impossible, d'évaluer nos collègues qui posaient leur candidature de seuil tout en refusant de nous impliquer dans le même processus ».

21 Entre-temps, les syndicats d'enseignants, affaiblis comme tous les syndicats britanniques après la législation mise en place par le gouvernement Thatcher, étaient bien plus occupés à s'assurer que le nouveau système était équitable et transparent qu'à s'opposer à la nouvelle approche, qui offrait des bénéfices tangibles à leurs membres au travers de la revalorisation de la grille salariale.

\section{Comment crée-t-on une régulation entre établissements?}

D'une certaine façon, le cycle de gestion des performances ne contribue pas directement à harmoniser les pratiques entre établissements, si ce n'est que le système est national et que la gestion et la formation sont centralisées en matière de définition des objectifs. Toutefois, il existe dans le système anglais de nombreux autres dispositifs de régulation chargés d'améliorer l'homogénéité de l'expérience éducative des élèves, dans le cadre de l'amélioration plus générale du système scolaire. Nous disposons de programmes nationaux (le National Curriculum) qui définissent les exigences de formation pour tous les 
élèves, auxquels s'ajoute une supervision spécifique pour l'enseignement de l'anglais et des mathématiques. En ce qui concerne les enseignants, nous disposons également de normes nationales définies par le gouvernement, comprenant différentes séries de compétences dont ceux-ci doivent faire preuve à certains stades de leur carrière et qui concernent aussi bien ceux qui viennent d'être nommés que ceux qui souhaitent devenir chefs d'établissement. Parce que ces objectifs sont liés aux projets de développement des établissements, qui sont à leur tour régis par les objectifs définis par chaque établissement et par le système national d'inspection (Ofsted ${ }^{7}$ ), il se crée inévitablement un alignement progressif des approches dans l'ensemble des établissements. En ce qui concerne les chefs d'établissement, l'implication des partenaires d'amélioration scolaire (ou SIP, School Improvement Partners) dans la définition des objectifs contribue également à mettre l'accent sur les priorités régionales et nationales, et donc à renforcer la façon dont l'ensemble des établissements fonctionne dans un cadre commun, vers un but commun. De surcroît, alors que la plupart des chefs d'établissement et des enseignants pensent que la publication des résultats d'examen et des palmarès d'établissements est inutile ou inefficace, il est évident que de tels palmarès obligent les établissements à faire aussi bien, sinon mieux, que leurs concurrents directs.

\section{Quelles transformations ce pilotage induit-il ?}

Selon mon témoin, ce nouveau système a incontestablement induit des changements, dont fort peu ont été négatifs. Les problèmes qu'il a fallu résoudre ont majoritairement porté sur les financements ou les détails pratiques. Il ne fait aucun doute qu'il est indispensable de consacrer un temps considérable à ce processus et que, par conséquent, on dispose de moins de temps pour d'autres tâches - y compris l'enseignement. Les coûts de fonctionnement sont pour partie dus au temps durant lequel les collègues n'enseignent pas, en raison de leur implication dans le processus de gestion des performances (qui comprend l'observation des cours) et aux besoins actuels de formation, tant pour les jeunes collègues qui embrassent la carrière que pour rafraîchir les compétences des enseignants expérimentés. Mes propres discussions informelles avec des collègues en activité mettent en évidence les questions de temps et d'horaires, et d'aucuns ne manqueront pas de prime abord de considérer ces activités comme une perte de temps pure et simple. Toutefois, lorsqu'on les pousse dans leurs retranchements, ils finissent par admettre le côté positif de la démarche. Selon eux, le problème réside davantage dans la gestion du temps, denrée rare dans un cadre professionnel surchargé.

On considère toutefois que les bénéfices qui résultent de la gestion des performances l'emportent largement sur les problèmes de coûts, en termes de temps et d'argent. Mon témoin confirme qu'il a observé - et continue d'observer - des améliorations significatives en matière d'enseignement et d'apprentissage. La transparence s'est considérablement accrue et les enseignants débattent de plus en plus de la manière d'améliorer la façon dont les élèves apprennent. Ces débats, qui ne surviennent pas seulement dans le cadre des réunions formelles planifiées mais aussi autour d'un café ou $\mathrm{du}$ distributeur de boissons, dénotent une amélioration du professionnalisme des enseignants et de la qualité du débat pédagogique. Ils embrassent un champ allant de la philosophie ou des grands principes au partage de pratiques pédagogiques concrètes et aux suggestions d'idées provenant de collègues. Les enseignants ont appris à admettre qu'ils ont eux aussi besoin d'aide dans certains domaines : « Tout ça a abattu les barrières 
et vaincu la peur du "Je ne veux pas qu'on voie ce que je fais dans ma classe". L'autre effet positif a été de permettre à certains de se libérer en admettant qu'un peu d'aide ne serait pas de trop. Ils finissent par reconnaître qu'ils ne parviennent pas toujours à faire face. Ce système leur a permis de découvrir que leurs collègues les encouragent au lieu de les juger, ce qui a été une vraie surprise. J'ai pu observer tout cela à l'œuvre dans les trois établissements que je connais. Incontestablement, c'est un progrès ».

La gestion des performances, et plus particulièrement les observations faites en classe qui font partie intégrante du système, a permis aux enseignants d'être plus réceptifs les uns aux autres et d'échanger des informations conduisant à une meilleure pratique professionnelle. Les portes des salles de classe anglaises sont vraisemblablement plus ouvertes aujourd'hui, au sens propre comme au sens figuré.

\section{Le mot de la fin : l'avis du chef d'établissement anglais}

26 À la fin de mon entretien avec mon chef d'établissement témoin, je lui ai demandé quels conseils il donnerait à ses collègues français, alors que ceux-ci s'apprêtent à mettre en place un nouveau système de gestion des performances. Après avoir insisté sur la nécessité de pouvoir disposer de suffisamment de temps et d'argent pour ce faire, il a déclaré que le système ne valait que par les gens qui le composent. L'enseignant évaluateur et l'enseignant évalué doivent comprendre comment donner et recevoir cette information en retour et comment négocier des objectifs SMART raisonnables.

Ses derniers mots, montrant à quel point il a été convaincu par l'introduction de ce système, parlent d'eux-mêmes et offrent une excellente conclusion à cet article. Je lui ai demandé : «Si le DfES modifiait sa politique demain et déclarait "Nous allons abandonner ce projet, nous n'en avons plus besoin”, que feriez-vous?». Il m'a répondu: « Nous continuerions. C'est plutôt intéressant de voir que nous n'y renoncerions pas, et je vous parie que de nombreux établissements feraient de même. Ils n'ont pas l'intention de laisser tomber. Tout au plus serait-il question d'alléger un peu les contraintes, mais ils veulent continuer parce que ça permet à chaque membre de l'équipe pédagogique de s'imprégner de l'ensemble du projet d'établissement au lieu de penser que ça ne concerne que le chef d'établissement ou les gestionnaires - tout le monde sauf lui, en somme. Pour moi, c'est l'argument le plus fort ».

\section{Remerciements}

Je suis extrêmement reconnaissante à l'académie de Versailles de m'avoir invitée pour une conférence, ce qui a fourni la matière de cet article. Je voudrais également remercier le $D^{r}$ Andrew Wright, directeur du Lincoln Christ's Hospital School, pour l'ouverture d'esprit dont il a fait preuve en m'autorisant à l'interviewer et à utiliser son expertise pour étayer ce travail.

BIBLIOGRAPHIE 


\section{Sources d'information complémentaires :}

Le système scolaire anglais est expliqué en détail sur le site Internet de l'Agence de formation et de développement des écoles (Training and Developement Agency for Schools) : http:// www.teachernet.gov.uk/management/payandperformance/performancemanagement/

Le cadre réglementaire ayant trait à la gestion des performances peut également être consulté sur : http://www.teachernet.gov.uk/docbank/index.cfm?id=10404

Les principaux syndicats enseignants proposent également des informations et des liens sur leurs sites :

National Association of Schoolmasters Union of Women Teachers I http://www.nasuwt.org.uk/

Templates/Internal.asp?NodeID=71742

National Union of Teachers : http://www.nut.org.uk/story.php?id=3974

\section{NOTES}

1. Cet article rend compte d'une présentation par Muriel Robinson de l'expérience anglaise du pilotage par objectifs organisée en mai 2007, à l'initiative de $\mathrm{M}^{\text {me }}$ Odile Luginbühl, IA-IPR de lettres modernes, devant des inspecteurs et chefs d'établissement français de l'académie de Versailles.

2. Chaque établissement anglais dispose d'un conseil d'administration constitué de membres volontaires issus de la communauté locale, comprenant des représentants des parents et des enseignants. Les membres de ce conseil sont responsables de tous les aspects de la vie scolaire, y compris du financement des programmes, du budget et des bâtiments. Dans la pratique, beaucoup de ces tâches sont conduites par des sous-comités spécifiques. (NdA)

3. NdA: ce schéma vient du TDA (Training and Development Agency for Schools, Agence gouvernementale pour la formation et le développement des établissements scolaires).

4. $\mathrm{NdT}$ : jeu de mots intraduisible sur l'adjectif smart, repris sous forme d'acronyme, dont le sens est ici « malin, intelligent ».

5. Ndlr : General Certificate of Secondary Education, examen passé à l'âge de 15 ans. Voir Cécile de Bouttemont: "Le système éducatif en Angleterre et au Pays de Galles ", Revue internationale d'éducation de Sèvres $n^{\circ} 200$.

6. Ndlr : Department for Education and Skills. (Département de l'Éducation et des compétences).

7. Ndlr: Office for Standards in Education, Children's Services and Skills (Bureau des normes en matière d'éducation, de services à l'enfant et de compétences).

\section{INDEX}

Mots-clés : politique éducative, système éducatif, système scolaire

Index géographique : Angleterre, Royaume-Uni 


\section{AUTEURS}

\section{MURIEL ROBINSON}

Professeur, directrice du Bishop Grosseteste University College, Royaume-Uni. 